\begin{tabular}{|c|l|}
\hline Title & Flat rotation curves in Chern-Simons modified gravity \\
\hline Author(s) & Konno, Kohkichi; Matsuyama, Toyoki; A sano, Y asuhiro; Tanda, Satoshi \\
\hline Citation & $\begin{array}{l}\text { Physical Review. D, Particle, fields, gravitation, and cosmology, 78(2), 024037 } \\
\text { https://doi.org/_0.1103/PhysRevD.78.024037 }\end{array}$ \\
\hline Issue Date & 2008_07 \\
\hline Doc URL & http://hdl.handle.net/2115/35017 \\
\hline Rights & ○ 2008The American Physical Society \\
\hline Type & article \\
\hline File Information & PhysRevD_78_024037.pdf \\
\hline
\end{tabular}

Instructions for use 


\title{
Flat rotation curves in Chern-Simons modified gravity
}

\author{
Kohkichi Konno, ${ }^{1, *}$ Toyoki Matsuyama, ${ }^{2}$ Yasuhiro Asano, ${ }^{1}$ and Satoshi Tanda ${ }^{1}$ \\ ${ }^{1}$ Department of Applied Physics, Hokkaido University, Sapporo 060-8628, Japan \\ ${ }^{2}$ Department of Physics, Nara University of Education, Nara 630-8528, Japan
}

(Received 5 October 2007; published 23 July 2008)

\begin{abstract}
We investigate the spacetime of a slowly rotating black hole in the Chern-Simons modified gravity. The long range feature of frame-dragging effect under the Chern-Simon gravity well explains the flat rotation curves of galaxies which is a central evidence of dark matter. Our solution provides a different scenario of rotating space from Gödel's solution.
\end{abstract}

DOI: 10.1103/PhysRevD.78.024037

PACS numbers: 04.50.- $\mathrm{h}, 04.70 . \mathrm{Bw}, 95.35 .+\mathrm{d}$

\section{INTRODUCTION}

There are three fundamental unsolved issues in the theory of gravity: quantization of gravity, dark energy, and dark matter. The string theory [1] is a promising candidate for a consistent quantum theory of gravity. Many attempts in quantizing gravity, however, have not been successful. In astronomy/astrophysics, a number of observations suggest the existence of dark energy $[2,3]$ and dark matter $[3,4]$. Although many surveys of astrophysical objects have been conducted [5], it has not yet been revealed what is dark matter. For instance, the flat rotation curves of galaxies [6] have been considered to be a robust evidence of dark matter. The velocity $v$ of a star orbiting around the center of a galaxy becomes a constant at a certain distance $r$ far from the center. While the Newtonian gravity yields a relation $v \propto 1 / \sqrt{r}$. At present, we usually attribute the discrepancy to dark matter. However it may be still possible to explain the phenomenon based on a theory without dark matter. In this paper, we propose a model to solve this discrepancy in the framework of the Chern-Simons (CS) gravity.

The CS action [see Eq. (1)]is a universal entity obtained from an effective action in the string theory [1,7]. In fact, the action also appears in condensed matter physics such as the quantum Hall effect [8,9]. Deser et al. [10] originally constructed a theory of CS gravity in $(2+1)$ dimensions and recently Jackiw and Pi extended it to $(3+1)$ dimensions [11]. Several important consequences of the CS action have been discussed in theories of gravity [7,1214]. In connection with this study, we summarize two important features of the CS gravity as follows. First, the Schwarzschild solution satisfies the field equation of CS gravity, which indicates that the CS theory meets a requirement of classical tests for general relativity [15]. Second, generally speaking, the CS term enhances angular velocity or angular momentum. In other words, the CS term rather modifies the gravitomagnetic part of the gravitational field than the gravitoelectric part [16]. These facts imply that CS

\footnotetext{
*konno@topology.coe.hokudai.ac.jp
}

gravity may behave as if dark matter exists, which motivated this study.

In this paper, we study a solution for a slowly rotating black hole in the CS gravity based on a previous work [13]. The frame-dragging effect turns out to be significantly large in a region far from the gravitational source. As a consequence, the obtained solution explains the flat rotation curves of a galaxy. By analyzing the frame-dragging effect on precession of a spinning object, we also discuss a way to confirm the CS gravity in observations. This paper indicates a route connecting the quantum theory of gravity with the dark matter problem. Hereafter, we use the geometrized units with $c=G=1$.

\section{CS GRAVITY}

We briefly review a theory of the CS modified gravity [11]. The action in this paper is given by the EinsteinHilbert action and the CS one,

$$
I=\frac{1}{16 \pi} \int d^{4} x\left(-\sqrt{-g} R+\frac{1}{4} l \vartheta^{*} R_{\tau^{\sigma \nu}}^{\mu \nu}{ }_{\sigma \mu \nu}\right),
$$

where $g$ is the determinant of the metric, $R \equiv g^{\alpha \beta} R_{\alpha \lambda \beta}^{\lambda}$ is the Ricci scalar, $R_{\sigma \alpha \beta}^{\tau} \equiv \partial_{\beta} \Gamma_{\sigma \alpha}^{\tau}-\cdots$ is the Riemann tensor ( $\Gamma_{\beta \gamma}^{\alpha}$ is the Christoffel symbols), $l$ is a coupling constant, and $\vartheta$ is an external quantity. The dual Riemann tensor density is defined by ${ }^{*} R^{\tau}{ }_{\sigma}{ }^{\mu \nu} \equiv \frac{1}{2} \varepsilon^{\mu \nu \alpha \beta} R_{\sigma \alpha \beta}^{\tau}$, where $\varepsilon^{\mu \nu \alpha \beta}$ is the Levi-Civita tensor density of weight one. The variation of the action with respect to $g_{\mu \nu}$ gives a field equation

$$
G^{\mu \nu}+l C^{\mu \nu}=-8 \pi T^{\mu \nu},
$$

where $G^{\mu \nu}$ is the Einstein tensor, $T^{\mu \nu}$ is the energymomentum tensor, and $C^{\mu \nu}$ is the Cotton tensor defined as

$$
\begin{aligned}
C^{\mu \nu}= & -\frac{1}{2 \sqrt{-g}}\left[v_{\sigma}\left(\varepsilon^{\sigma \mu \alpha \beta} \nabla_{\alpha} R_{\beta}^{\nu}+\varepsilon^{\sigma \nu \alpha \beta} \nabla_{\alpha} R_{\beta}^{\mu}\right)\right. \\
& \left.+\left(\nabla_{\sigma} v_{\tau}\right)\left({ }^{*} R^{\tau \mu \sigma \nu}+{ }^{*} R^{\tau \nu \sigma \mu}\right)\right] .
\end{aligned}
$$

Here, $v_{\mu} \equiv \partial_{\mu} \vartheta$ is called an embedding vector. In this theory, the condition 


$$
0=\nabla_{\mu} C^{\mu \nu}=\frac{1}{8 \sqrt{-g}} v^{\nu *} R_{\tau}^{\sigma}{ }^{\mu \lambda} R_{\sigma \mu \lambda}^{\tau}
$$

should be imposed to ensure the diffeomorphism invariance. This condition is derived by using the Bianchi identity $\nabla_{\mu} G^{\mu \nu}=0$ and the equation of motion $\nabla_{\mu} T^{\mu \nu}=0$. Equations (2) and (4) are basic equations in the CS gravity.

In the previous work [13], we obtained a solution of Eqs. (2) and (4) for a slowly rotating black hole by using the perturbation expansion around the Schwarzschild solution. In this paper, we particularly concentrate our attention on the case of a spacelike vector $v_{\mu}=\partial_{\mu} \vartheta=\partial_{\mu} z=\partial_{\mu}(r \cos \theta)=(0, \cos \theta,-r \sin \theta, 0)$.

The metric is provided by

$$
\begin{aligned}
d s^{2}= & -\left(1-\frac{2 M}{r}\right) d t^{2}+\left(1-\frac{2 M}{r}\right)^{-1} d r^{2} \\
& +r^{2}\left(d \theta^{2}+\sin ^{2} \theta d \phi^{2}\right)-2 r^{2} \omega(r) d t d \phi,
\end{aligned}
$$

where $M$ is mass of a black hole, the $\theta$ dependence of the $(t \phi)$ component is restricted by Eq. (4), and

$$
\begin{aligned}
\omega= & \frac{C_{1}}{r^{2}}\left(1-\frac{2 M}{r}\right)+\frac{C_{2}}{r^{3}}\left[r^{2}-2 M r-4 M^{2}\right. \\
& +4 M(r-2 M) \ln (r-2 M)] .
\end{aligned}
$$

Here, $C_{1}$ and $C_{2}$ are constants characterizing the rotation of a black hole and are related to a small parameter $\epsilon(\equiv$ $J / M r)$, i.e., $C_{1}, C_{2} \sim O(\epsilon)$, where $J$ is the angular momentum. This solution satisfies both Eqs. (2) and (4) up to the first order in $\epsilon$. Within the first order of $\epsilon$, the solution does not depend on $l$. The differential equation for $\omega$ can be obtained from the nonvanishing components of the field equation. In the $(t \phi)$ component, the Einstein tensor gives the differential equation, whereas the Cotton tensor vanishes. In contrast in other components, the Einstein tensor vanishes, and the Cotton tensor gives the same differential equation. Therefore, Eq. (6) is also a solution in general relativity. We emphasis that the Kerr solution does not satisfy Eq. (4), which is an important difference between the Einstein gravity and the CS gravity.

Here we briefly mention a relation between the original CS gravity and the string theory. In the original CS gravity, Eq. (4) is imposed to ensure the diffeomorphism invariance. As shown in Ref. [7], $\vartheta$ in Eq. (1) is not an external quantity but a dynamical variable in the framework of string theory. Therefore Eq. (4) is replaced by the field equation [7]

$$
g^{\mu \nu} \nabla_{\mu} \nabla_{\nu} \vartheta=-\frac{l}{64 \pi \sqrt{-g}}{ }^{*} R_{\tau}^{\sigma}{ }^{\mu \nu} R_{\sigma \mu \nu}^{\tau}
$$

in the string theory. When $l$ is a nonvanishing value and $\vartheta$ is a constant, Eqs. (2) and (7), respectively, reduce to $G^{\mu \nu}=0$ and ${ }^{*} R^{\sigma}{ }_{\tau}^{\mu \lambda} R_{\sigma \mu \lambda}^{\tau}=0$ in a vacuum. We emphasis that our solution in Eqs. (5) and (6) also satisfies these equations. Thus Eqs. (5) and (6) describe a classical field which includes effects of quantum gravity. Furthermore, we need not to align the rotational axis with the embedding vector in this case because of $v^{\mu}=0$. Hereafter we consider Eq. (7) rather than Eq. (4) because Eq. (7) makes the CS theory self-consistent.

\section{FEATURES OF SOLUTION}

We look into a solution of the spacetime of a slowly rotating black hole. For this purpose, we consider a scalar invariant $R^{\alpha \beta \gamma \delta} R_{\alpha \beta \gamma \delta}$ which is useful to evaluate the radial dependence of the frame-dragging effect. From the metric, the scalar invariant at large $r$ is obtained in the form

$$
\begin{aligned}
R^{\alpha \beta \gamma \delta} R_{\alpha \beta \gamma \delta}= & \left(R^{(0) \alpha \beta \gamma \delta}+R^{(1) \alpha \beta \gamma \delta}\right)\left(R_{\alpha \beta \gamma \delta}^{(0)}\right. \\
& \left.+R^{(1)}{ }_{\alpha \beta \gamma \delta}\right) \\
\simeq & \frac{48 M^{2}}{r^{6}}-\frac{4 C_{2}^{2}}{r^{4} \sin ^{4} \theta},
\end{aligned}
$$

where "(0)" and "(1)" denote the zeroth and the first order in $\epsilon$, respectively. The first term stems from the Schwarzschild solution, and the second term corresponds to the rotation of a black hole. For $\theta \neq 0, \pi$, the scalar invariant asymptotically reduces to zero at large $r$. As a consequence, the spacetime becomes asymptotically flat at infinity. The singularity of the rotational axis could be avoided by finding nonlinear or exact solutions. We note that the frame-dragging part proportional to $r^{-4}$ decays more slowly than the Schwarzschild part proportional to $r^{-6}$. On the derivation of our metric solution we use the absence of the Chern-Pontryagin density ${ }^{*} R^{\sigma}{ }_{\tau}^{\mu \nu} R^{\tau}{ }_{\sigma \mu \nu}$ which is a source of gravitational anomaly [17]. Thus the absence of gravitational anomaly gives the long range nature of the frame-dragging effect. For comparison, we recall the Kerr metric whose $(t \phi)$ component at large $r$ is given by $g_{t \phi}^{(\mathrm{K})}=-2 J_{0} \sin ^{2} \theta / r$, where $J_{0} \sim O(\epsilon)$ is the angular momentum. For this metric, we obtain

$$
R_{(\mathrm{K})}{ }^{\alpha \beta \gamma \delta} R_{(\mathrm{K}) \alpha \beta \gamma \delta} \simeq \frac{48 M^{2}}{r^{6}}-\frac{144 J_{0}^{2}}{r^{8}}(2+\cos 2 \theta) .
$$

Thus the Kerr metric gives rise to the rapid decay of the frame-dragging part because the second term is proportional to $r^{-8}$.

\section{TRAJECTORIES OF A TEST PARTICLE}

To show more astrophysical consequences of the solution, we investigate trajectories of a test particle with a mass of $m$. Since the metric does not depend on $t$ and $\phi$, the $t$ and $\phi$ components of four-momentum $p_{\mu}$ are conserved for the particle [18]. Hence, it can be assumed that $p_{0}=-m E$ and $p_{\phi}=m L$, where $E$ and $L$ are the energy and angular momentum of the particle, respectively. We focus on trajectories in the equatorial plane $(\theta=\pi / 2)$. From $g_{\mu \nu} p^{\mu} p^{\nu}=-m^{2}$, we obtain 


$$
\left(\frac{d r}{d \tau}\right)^{2}=E^{2}-\left(1-\frac{2 M}{r}\right)\left(1+\frac{L^{2}}{r^{2}}\right)-2 E L \omega(r),
$$

where $\tau$ is a proper time. Differentiating this equation with respect to $\tau$, we derive

$$
\frac{d^{2} r}{d \tau^{2}}=-\frac{1}{2} \frac{d}{d r}\left[\left(1-\frac{2 M}{r}\right)\left(1+\frac{L^{2}}{r^{2}}\right)\right]-E L \omega^{\prime}(r),
$$

with $\omega^{\prime} \equiv d \omega / d r$. These equations can be solved perturbatively because the last terms on the right-hand side of Eqs. (10) and (11) are small compared with the other terms, i.e., $\omega, \omega^{\prime} \sim O(\epsilon)$.

We solve Eqs. (10) and (11) up to the first order in $\epsilon$ for circular orbits $(r=$ const). The solutions are then given by

$$
\begin{aligned}
E= & \frac{r-2 M}{\sqrt{r(r-3 M)}} \\
& \pm \sqrt{\frac{M}{r-3 M}}\left[r \omega(r)+\frac{r^{2}(r-2 M)}{2(r-3 M)} \omega^{\prime}(r)\right], \\
L & = \pm r \sqrt{\frac{M}{r-3 M}}+\frac{r^{4}(r-2 M)}{2 \sqrt{r(r-3 M)^{3}}} \omega^{\prime}(r) .
\end{aligned}
$$

Using these quantities, we can calculate $d \phi / d t=$ $(d t / d \tau)^{-1} d \phi / d \tau$ for circular orbits. The circular velocity is then obtained as

$$
v=r \frac{d \phi}{d t}= \pm \sqrt{\frac{M}{r}}+\left[r \omega(r)+\frac{r^{2}}{2} \omega^{\prime}(r)\right] .
$$

This is the central result of this paper. The first term, which is a monotonically decreasing function proportional to $r^{-1 / 2}$, is coming from the Schwarzschild metric. It is very surprising that the second term becomes a constant at large $r$, i.e.,

$$
v \simeq \pm \sqrt{\frac{M}{r}}+\frac{C_{2}}{2} .
$$

Therefore, the same feature can be expected in the rotation curves of galaxies. On the other hand in the case of the Kerr solution, the frame-dragging part is negligible at large $r$ because $v_{(\mathrm{K})} \simeq \pm \sqrt{M / r}-J_{0} / r^{2}$ is derived. In a galaxy, there are a bulge and a disk associated with the central black hole. For the spherically symmetric part such as a bulge, an outside spacetime solution has the same form as that of our solution. The nonspherical part such as a disk would deform the solution in the direction of the rotational axis. Thus the feature of the flat rotation curve is considered to remain unchanged on the equatorial plane.

At first glance, the constant circular velocity $v$ at infinity seems to contradict the asymptotically flat spacetime. This, however, is explained as follows. It is impossible to cover the spatial infinity by a single Minkowski spacetime. To do so, a number of Minkowski spacetimes are necessary. It is possible to consider that the spatial infinity is covered by several finite-size areas and that each area is covered by a Minkowski spacetime. In such a situation, two adjacent areas are smoothly connected with each other by virtue of the infinitesimal curvature which is given by the second term of Eq. (8). This situation is similar to that for a vector potential field created by a solenoid with magnetic flux $\Phi$. For a certain gauge, the vector potential has the form $A_{i}=$ $(\Phi / 2 \pi)\left(-y /\left(x^{2}+y^{2}\right), x /\left(x^{2}+y^{2}\right), 0\right)$, where $i$ represents spatial indexes. [This is very similar to Eq. (17) below.] If the exact form $A_{i} d x^{i} \equiv d a$ is considered, any function for $a$ cannot cover the whole space. Because of this fact, the loop integral of $A_{i}$ can give the nonzero value $\Phi$, i.e., $\oint A_{i} d x^{i}=\oint d a=\Phi$.

The angular momentum of the Kerr black hole does not depend on choices of a two-surface within the definition of $J=\int \sqrt{-g} \varepsilon_{\alpha \beta \theta \phi} \nabla^{\alpha} \psi^{\beta} d \theta d \phi / 16 \pi$ [19], where $\psi^{\mu}=$ $(0,0,0,1)$ is the Killing vector. When we evaluate the angular momentum of a black hole in Eqs. (5) and (6) by using the same definition, the angular momentum depends not only on $C_{1}$ and $C_{2}$ but also on $r$. This means that the gravitational field also has the angular momentum whose degree is given by $C_{1}$ and $C_{2}$. It should also be noted that such a situation is a result of $\vartheta=$ const in Eq. (7). When $\vartheta$ is changed from a constant at large $r$, the frame-dragging effect would also be modified. In particular, when ${ }^{*} R^{\sigma}{ }_{\tau}{ }^{\mu \lambda} R_{\sigma \mu \lambda}^{\tau} \neq 0$ in Eq. (7), the solution of Eq. (2) may have the same feature as that of the Kerr solution.

\section{PRECESSION OF SPINNING OBJECTS}

We discuss a way to confirm the CS gravity through the precession of spinning objects. To discuss the precession of spinning objects, we first adopt the isotropic form of the metric derived from the transformation, $(x, y, z)=$ $(\tilde{r} \sin \theta \cos \phi, \tilde{r} \sin \theta \sin \phi, \tilde{r} \cos \theta), \quad$ where $\quad r=\tilde{r}(1+$ $M / 2 \tilde{r})^{2}$. Then we apply the weak field approximation to discuss more accessible situations. Namely the postNewtonian approximation is applied to the diagonal components of the metric and the leading term at large $r$ is considered in the off diagonal components $g_{t i}$. We obtain

$$
\begin{aligned}
d s^{2} \simeq & -(1-2 U) d t^{2}+(1+2 U)\left(d x^{2}+d y^{2}+d z^{2}\right) \\
& +2 N_{i}^{(1)}(x, y, z) d x^{i} d t
\end{aligned}
$$

where $U=M / r$, and $N_{i}^{(1)}$ is given by

$$
N_{i}^{(1)}=\left(C_{2} \frac{y \tilde{r}}{x^{2}+y^{2}},-C_{2} \frac{x \tilde{r}}{x^{2}+y^{2}}, 0\right) .
$$

By using the Minkowski metric $\eta_{\mu \nu}$, we define $h_{\mu \nu} \equiv$ $g_{\mu \nu}-\eta_{\mu \nu}$. It is shown that $h_{\mu \nu}$ satisfies the Lorentz gauge condition $\partial_{\alpha} h^{\alpha}{ }_{\mu}-\partial_{\mu} h_{\lambda}^{\lambda} / 2=0$ under Eq. (16). In a local Lorentz frame momentarily comoving with a spin- 
ning object, the spin obeys an equation $d S_{(i)} / d \tau=$ $S_{\mu} u^{\nu} \nabla_{\nu} e_{(i)}^{\mu}$, where $e_{(\alpha)}^{\mu}$ denotes the tetrad and $S_{(i)}=$ $e_{(i)}^{\mu} S_{\mu}$ is the spin vector [15]. The four velocity of the spinning object $u^{\mu}=e_{(0)}^{\mu}$ is approximated as $u^{\mu} \simeq$ $\left(1, v^{k}\right)$. In the weak field approximation in Eq, (16), the equation becomes $d \boldsymbol{S} / d \tau=\boldsymbol{\Omega} \times \boldsymbol{S}$, where $\boldsymbol{\Omega}=-(\boldsymbol{v} \times$ $\boldsymbol{a}) / 2+3(\boldsymbol{v} \times \nabla U) / 2-\left(\nabla \times \boldsymbol{N}^{(1)}\right) / 2$ and $\boldsymbol{a}$ denotes the acceleration vector. This equation seems to have the same form as that obtained under the Kerr solution. The last term of $\boldsymbol{\Omega}$, however, has a different form. We obtain

$$
\mathbf{\Omega}_{N}=\left(-\frac{C_{2}}{2} \frac{x z}{\left(x^{2}+y^{2}\right) \tilde{r}},-\frac{C_{2}}{2} \frac{y z}{\left(x^{2}+y^{2}\right) \tilde{r}}, \frac{C_{2}}{2 \tilde{r}}\right)
$$

for our solution and

$$
\boldsymbol{\Omega}_{N}^{(\mathrm{K})} \simeq\left(\frac{3 J_{0} x z}{\tilde{r}^{5}}, \frac{3 J_{0} y z}{\tilde{r}^{5}}, \frac{J_{0}\left[2 z^{2}-\left(x^{2}+y^{2}\right)\right]}{\tilde{r}^{5}}\right),
$$

for the Kerr solution. For large $r$, Eq. (18) is proportional to $r^{-1}$, whereas Eq. (19) decays as $r^{-3}$. This qualitative difference can be measured from the radial dependence of the spin precession. For such verification, observations should be done for spinning objects far from a black hole. Thus $C_{2}$ can be estimated from observed data according to Eq. (18).

\section{DISCUSSION}

The whole universe given by Eqs. (5) and (6) slightly rotates. However, it is difficult to observe the rotation at infinity because the angular displacement of objects decreases proportional to $1 / r$ with increasing $r$. The behavior of our solution at infinity is in contrast to Gödel's solution [20] in the Einstein theory. Equations (5) and (6) also mean that a galaxy is affected by the frame-dragging pull of another galaxy. Thus we should observe the correlation between the rotational velocity in a galaxy and the peculiar velocities of other galaxies to confirm the frame-dragging effect. It would be possible to estimate $C_{2}$ from analysis of the correlation. By now, unfortunately, a few studies have been made on this correlation. The frame-dragging effect might explain another evidences of dark matter, i.e., large velocity dispersion of galaxies in clusters [4]. The velocity dispersion in interacting galaxies may be expected to be larger than that in the Newtonian picture. Future investigation for cosmological density perturbation in this direction is necessary.

We remark the difference between our theory and the other modified gravity theories. The modified Newtonian dynamics [21] certainly describe the flat rotation curves. However, The modified Newtonian dynamics are entirely phenomenological and not supported by a recent experiment [22]. Similarly, the $f(R)$ gravity is phenomenological in practice [23]. On the other hand, the CS gravity is directly related to an essential part in the string theory, and our results are basically parameter free. Therefore, our theory should be distinguished from the other theories.

\section{CONCLUSION}

We have investigated features of the spacetime endowed with a slowly rotating black hole within the framework of the Chern-Simons (CS) gravity. We found that the CS gravity enhances the gravitomagnetic part of the gravitational field far from a black hole. As a consequence, the velocity of a test particle in a circular orbit surprisingly becomes a constant far from a black hole as it was found in a galaxy's rotation curve. Thus the result explains a robust evidence of dark matter without introducing realistic matter. Our finding indicates a possibility to solve the dark matter problem by a new theoretical framework of the CS gravity. To confirm the validity of this approach, we need to explain other evidences of dark matter such as large velocity dispersion of galaxies in cluster and structure formation in the Universe. Further investigation in this direction could bridge the gap between purely theoretical quantum gravity and more realistic astrophysical phenomena.

\section{ACKNOWLEDGMENTS}

This work was supported in part by a Grant-in-Aid for Scientific Research from The 21st Century COE Program "Topological Science and Technology." We thank Dr. K. Hayasaki for useful discussions. One author (K. K.) thanks Professor T. Futamase for stimulating comments. Analytical calculations were performed in part on computers at YITP at Kyoto University.
[1] J. Polchinski, String Theory (Cambridge University Press, Cambridge, 1998).

[2] A. G. Riess et al., Astron. J. 116, 1009 (1998).

[3] C.L. Bennett et al., Astrophys. J. Suppl. Ser. 148, 1 (2003).

[4] V. Trimble, Annu. Rev. Astron. Astrophys. 25, 425 (1987).

[5] B. R. Oppenheimer et al., Science 292, 698 (2001); A. J.
Romanowsky et al., Science 301, 1696 (2003); R. Massey et al., Nature (London) 445, 286 (2007).

[6] V. C. Rubin, Science 220, 1339 (1983).

[7] T.L. Smith, A. L. Erickcek, R. R. Caldwell, and M. Kamionkowski, Phys. Rev. D 77, 024015 (2008).

[8] R. Jackiw, Phys. Rev. D 29, 2375 (1984).

[9] T. Matsuyama, Prog. Theor. Phys. 77, 711 (1987). 


\section{FLAT ROTATION CURVES IN CHERN-SIMONS ...}

[10] S. Deser, R. Jackiw, and S. Templeton, Ann. Phys. (N.Y.) 140, 372 (1982).

[11] R. Jackiw and S.-Y. Pi, Phys. Rev. D 68, 104012 (2003).

[12] S. Alexander and N. Yunes, Phys. Rev. D 75, 124022 (2007); Phys. Rev. Lett. 99, 241101 (2007).

[13] K. Konno, T. Matsuyama, and S. Tanda, Phys. Rev. D 76, 024009 (2007).

[14] D. Guarrera and A. J. Hariton, Phys. Rev. D 76, 044011 (2007).

[15] C.M. Will, Theory and Experiment in Gravitational Physics (Cambridge University Press, Cambridge, 1993), 2nd ed..

[16] K. A. Moussa, G. Clement, and C. Leygnac, Classical Quantum Gravity 20, L277 (2003).
PHYSICAL REVIEW D 78, 024037 (2008)

[17] T. Kimura, Prog. Theor. Phys. 42, 1191 (1969); L. Alvarez-Gaumé and E. Witten, Nucl. Phys. B234, 269 (1984).

[18] B.F. Schutz, A First Course in General Relativity (Cambridge University Press, Cambridge, 1985).

[19] R. M. Wald, General Relativity (University of Chicago Press, Chicago, 1984).

[20] K. Gödel, Rev. Mod. Phys. 21, 447 (1949).

[21] M. Milgrom, Astrophys. J. 270, 365 (1983); 270, 371 (1983); 270, 384 (1983).

[22] J. H. Gundlach et al., Phys. Rev. Lett. 98, 150801 (2007).

[23] C. Frigerio Martins and P. Salucci, Mon. Not. R. Astron. Soc. 381, 1103 (2007). 